\title{
CUESTIONARIOS DE ATENCIÓN ESPIRITUAL EN CUIDADOS PALIATIVOS: REVISIÓN DE LA EVIDENCIA PARA SU APLICACIÓN CLÍNICA
}

\section{QUESTIONNAIRES ON SPIRITUAL CARE IN PALLIATIVE CARE: OVERVIEW OF THEIR CLINICAL APPLICABILITY}

Laura Galiana', Amparo Oliver², Enric Benito², Noemí Sansó3

' Departamento de Metodología de las Ciencias del Comportamiento de la Universitat de València. Av. Blasco Ibáñez, 21, 46010, Valencia, España

2 Enric Benito. Consultor senior en Cuidados Paliativos.

${ }^{3}$ Departamento de Enfermería y Fisioterapia de la Universidad de las Islas Baleares. Cta. Valldemossa 7.5 Km. Palma de Mallorca, 07122, Mallorca, España

Resumen

Objetivo: Revisar los cuestionarios de evaluación de necesidades y recursos espirituales en cuidados paliativos más recientes y evaluar su aplicabilidad clínica en nuestro entorno.

Método: Revisión sistemática, siguiendo las guías PRISMA de las publicaciones realizadas durante 2015-2016 de trabajos sobre espiritualidad. Los artículos debían estar centrados en el cuidado espiritual o la evaluación de la espiritualidad, basados en población de cuidados paliativos; y sometidos a revisión por pares.

Resultado: de los 42 artículos identificados, tras evaluación independiente de dos observadores, 15 cumplieron criterios de selección. Estos se analizan respecto sus características, propiedades psicométricas y aplicabilidad.

Conclusiones: La necesidad de mejorar la atención espiritual en el proceso de morir ha Ilevado a la aparición de múltiples herramientas de evaluación y acompañamiento espiritual. Su uso debe ponderarse con la experiencia del clínico y su aplicabilidad según el entorno cultural en el que se ha construido y sus propiedades psicométricas

Palabras clave: Cuidados Paliativos, espiritualidad, cuestionarios, propiedades psicométricas, acompañamiento espiritual.
Abstract

Objective: review of the more recent questionnaires assessing spiritual needs and resources in palliative care, and determine their clinical applicability in our environment.

Methods: systematic review, following PRISMA guidelines, of all publications about spirituality appeared on 2015-2016. Papers had to be focused on spiritual care or spirituality assessment, based on a palliative care population, and gone through a peer review.

Results: a total of 42 publications were identified, but after the evaluation of two independent observers, only 15 fulfilled the selection criteria. The selected papers were analyzed according to their characteristics, psychometric properties and applicability.

Conclusions: the need of improving spiritual care in the dying process lead to the advent of many assessment and spiritual care tools. Their use has to be considered together with the clinician experience, their applicability on the cultural context and their psychometric properties.

Keywords: Palliative care, spirituality, outcome measures, psychometric properties, spiritual care.

\section{Correspondencia:}

Noemí Sansó.

Departamento de Enfermería y Fisioterapia de la Universidad de las Islas Baleares.

Cta. Valldemossa 7.5 Km. Palma de Mallorca, 07122, Mallorca (España).

E-mail: noemi.sanso@uib.es 


\section{PLANTEAMIENTO DEL PROBLEMA}

En las últimas dos décadas, la espiritualidad ha sido reconocida por investigadores, clínicos, pacientes y familiares como un recurso importante para hacer frente al distrés que acompaña la enfermedad terminal y el proceso de morir ${ }^{(1,2)}$. La literatura científica sobre espiritualidad en clínica, y más específicamente en el contexto de la oncología y los cuidados paliativos, ha aumentado exponencialmente y las guías nacionales e internacionales como el $\mathrm{Na}$ tional Consensus Project for Quality Palliative Care de 2011 (NCPQPC) ${ }^{(3)}$, el National Quality Forum ${ }^{(4)}$ o las publicaciones de la Sociedad Española de Cuidados Paliativos $(\text { SECPAL) })^{(5)}$ y de la Sociedad Europea de Cuidados Paliativos (EAPC) ${ }^{(6)}$, han consolidado la evidencia de que el cuidado espiritual es un componente imprescindible en la atención sanitaria de calidad en esta etapa de la vida ${ }^{(7)}$. En esta línea, la National Comprehensive Cancer Network, de $2003^{(8)}$ reconoce, en sus guías de manejo del distrés en la práctica clínica, la necesidad de identificar las preocupaciones espirituales como una fuente específica de distrés. También las guías para la práctica clínica del NCPQPC ${ }^{(3)}$, reconocen los aspectos espirituales, religiosos y existenciales del cuidado como uno de los ocho dominios del cuidado paliativo óptimo. Más recientemente el esfuerzo por incluir la dimensión espiritual en la práctica clínica, condujo a la elaboración de un documento de consenso internacional a través de una conferencia de expertos en Ginebra en $2013^{(7)}$.

Estos grupos y sus documentos han aportado distintas definiciones de la espiritualidad y lo que implica el cuidado espiritual. Según el NCPQPC ${ }^{(3)}$, la espiritualidad se define como el aspecto de la humanidad referido a la forma en que los individuos buscan y expresan significado y propósito y la forma en que experimen- tan su conexión con el momento, el yo, los otros, la naturaleza y lo sagrado ${ }^{(9)}$. En nuestro contexto, la Guía de Espiritualidad de la Sociedad Española de Cuidados Paliativos ${ }^{(10)}$, define la espiritualidad como la aspiración profunda e íntima del ser humano, el anhelo de una visión de la vida y la realidad que integre, conecte, trascienda y dé sentido a la existencia. Se asocia también al desarrollo de unas cualidades y valores que fomenten el amor y la paz.

Cada vez disponemos de más evidencia de cómo los pacientes con enfermedades que amenazan la vida y aquellos que se encuentran en las últimas etapas de la enfermedad terminal, pueden sentir un deseo mayor de acceder y enriquecer la parte espiritual de sus vidas. Según la National Comprehensive Cancer Network, el apoyo espiritual se ha relacionado con una mayor satisfacción del paciente con la atención médica ${ }^{(8)}$. Se ha estimado que entre el 86 y el $91 \%$ de los pacientes con cáncer avanzado tienen necesidades espirituales, que tradicionalmente se definen como búsqueda espiritual (i.e., búsqueda de perdón y reconciliación) o búsqueda de propósito y significado en la vida ${ }^{(11,12)}$. Un mayor bienestar espiritual se ha relacionado con mayor calidad de vida, funcionamiento psicosocial y menor depresión y ansiedad ${ }^{(13,-20)}$. Por el contrario, las necesidades espirituales no cubiertas, se han relacionado con una disminución de la calidad de vida, menor uso de atención paliativa y mayores costes de los cuidados de los pacientes paliativos ${ }^{(12,13,21,22)}$. En el contexto de la oncología, las creencias, comportamientos y experiencias espirituales pueden resultar recursos beneficiosos para el manejo de los retos físicos, mentales y sociales de la experiencia del cáncer. Por ejemplo, las experiencias espirituales del día a día se han asociado con mayor seguridad y menor miedo a la recurrencia del cáncer ${ }^{(23,24)}$. Por todo ello, parece razonable que la atención y el cuidado de 
los pacientes con enfermedad avanzada y terminal incluyan la evaluación de necesidades y recursos espirituales y su acompañamiento.

Hay un creciente consenso en la necesidad de procurar una atención a la dimensión espiritual en cuidados paliativos, y se han realizado diferentes propuestas para dicha evaluación en los últimos años ${ }^{(5)}$. Por ejemplo, The Joint Commission on Accreditation of Healthcare Organizations explica la necesidad de la evaluación del cuidado espiritual de todos los pacientes ${ }^{(25)}$. En algunos estudios realizados en los EEUU, con un entorno cultural muy diferente al español, los profesionales reconocen la importancia de identificar los recursos y necesidades espirituales de los pacientes de cuidados paliativos y se cita, que al menos un $40 \%$ de ellos, exploran estos recursos y necesidades en sus pacientes ${ }^{(26)}$. En nuestro país, un estudio reciente en profesionales de cuidados paliativos especialmente interesados en la atención espiritual, es decir una población muy sesgada a favor de este aspecto, muestra como el $94,2 \%$ consideró que el acompañamiento espiritual se encontraba dentro de las tareas propias de su profesión. Sin embargo, reconocían que no disponían de una documentación específica para explorarla $(59,2 \%)$ y sólo el $45 \%$ expresaba que la dimensión espiritual era considerada en su equipo. Este estudio señalaba la falta de preparación de los miembros de los equipos para el acompañamiento espiritual $(67 \%)$, así como formación específica muy escasa ${ }^{(27)}$.

Este interés en la atención espiritual y la necesidad de dotarnos de recursos para ello, ha llevado al desarrollo de gran variedad de instrumentos específicos. Actualmente, es frecuente la publicación de nuevos cuestionarios de evaluación y acompañamiento espiritual, aplicados en distintas poblaciones y nuevas aproximaciones a la espiritualidad. De hecho, en una revisión de los trabajos llevados a cabo durante el año 2014, se identificaron 6 estudios dirigidos a estudiar las propiedades psicométricas de nuevas y ya conocidas escalas de medida de la espiritualidad, 3 revisiones sobre la medida de la espiritualidad o aspectos relacionados, 3 trabajos cuyo objetivo fue consensuar la definición del cuidado espiritual y evaluación de la espiritualidad, y otros 3 trabajos centrados en temáticas relacionadas ${ }^{(28)}$. Sin embargo, y a pesar de esta necesidad de evaluación, pocos cuestionarios han sido evaluados en el contexto de la enfermedad avanzada ${ }^{(29)}$, y las que sí lo han hecho, cuentan con diversas limitaciones. Desde una perspectiva psicométrica, algunos resultados son poco concluyentes, debido a los retos que supone la evaluación de un constructo como la espiritualidad. De esta forma, aunque se han utilizado gran variedad de cuestionarios de espiritualidad en la literatura científica, muchas de ellas tienen pobres propiedades psicométricas ${ }^{(30,31)}$.

En este contexto, el objetivo de la presente investigación es resumir la evidencia actual sobre estas herramientas de evaluación de la atención y acompañamiento espiritual, con especial atención a las validaciones recientes y los nuevos instrumentos, las revisiones sobre la evaluación de los recursos espirituales y las propuestas de mejora para dicha evaluación.

\section{DESARROLLO DEL TEMA}

Para revisar los resultados más recientes identificados en la literatura de cuidados paliativos, se llevó a cabo una revisión sistemática, siguiendo las guías PRISMA (Preferred Reporting Items for Systematic Reviews and Meta-Analyses) ${ }^{(32)}$. Los pasos y criterios seguidos replican el estudio Ilevado a cabo por Oliver et al. ${ }^{(28)}$, actualizando los resultados de las aportaciones realizadas durante los años 2015-2016. 
La revisión siguió diferentes pasos. En primer lugar, se utilizaron diversos criterios para la búsqueda en las bases de datos. Posteriormente se seleccionaron los trabajos que versaron sobre la evaluación de la espiritualidad, utilizando diversos criterios: los artículos debían estar centrados en el cuidado espiritual o la evaluación de la espiritualidad, o al menos incluir una reflexión sobre estos temas; debían estar centrados en población de cuidados paliativos; y debían de estar sometidos a revisión por pares. Tras la búsqueda bibliográfica, se incluyeron a la revisión algunas contribuciones clave a partir de recomendaciones de expertos. Finalmente, se hizo acopio de las ideas principales, que se presentan de forma breve en estas páginas.

Siguiendo los pasos de Oliver et al.(28), se buscó en 22 bases de datos incluidas en la metabase de datos ProQuest Central. Para localizar artículos adicionales, se utilizó el motor de búsqueda Google Académico. La estrategia incluyó tres grupos de términos combinados con AND: "spiritual", and "palliative care" (inluyendo "end of life"), y "outcome" (incluyendo "measure"). La búsqueda se limitó a los trabajos publicados en 2015 y 2016, dado que los trabajos publicados en 2014 habían sido recogidos por Oliver et al. ${ }^{(28)}$. Los artículos encontrados fueron revisados de forma independiente por dos de las autoras, para verificar el cumplimiento de los criterios de inclusión y seleccionar aquellos relevantes para este trabajo.

La búsqueda permitió la identificación de 42 trabajos. Tras identificar aquellos artículos duplicados o que no habían sido revisados por pares, 15 de los trabajos fueron excluidos. A partir de la lectura de los textos, se excluyeron otros 13 trabajos de la revisión, por no estar centrados en la espiritualidad ( $n=9$ ), no tratarse de artículos científicos $(n=1)$ o no centrarse en población de cuidados paliativos $(\mathrm{n}=$ 3). Finalmente, se añadió un manuscrito a revisar a partir de las indicaciones de expertos, quedando un total de 15 artículos, que son específicamente indicados en la bibliografía y pueden consultarse en la tabla 1. Con los 15 artículos seleccionados, dos de las autoras realizaron la evaluación de forma independiente. En la puesta en común de los resultados se obtuvo un índice de Kappa estadísticamente significativo y elevado ( $\kappa=0,779, p=<0,001)$, lo que apuntó que la concordancia entre ambos criterios fue buena. Un breve resumen del proceso de revisión puede consultarse en la figura 1.

Como se puede observar en la figura 1, un primer grupo de cuatro artículos se centraron en la validación y el desarrollo de nuevos instrumentos. El trabajo de Masso et al. ${ }^{(33)}$, por ejemplo, presenta una nueva herramienta de medida, la Palliative Care Problem Severity Score, basada en la puntuación de los clínicos en cuatro dominios: dolor, otros síntomas, psicológico/espiritual y problemas de la familia/cuidadores. Se trata de una herramienta simple para evaluar la espiritualidad, que tiene como principal ventaja su brevedad y que sirve como un primer screening a partir del cual detectar si hay problemas psicológicos y/o espirituales y, por tanto, proceder a otras evaluaciones más precisas. La severidad del problema puede ser ausente, leve, moderada o severa. Sin embargo, los autores no ofrecen pautas para conocer a partir de qué grado sería necesaria una mejor evaluación y posible intervención posterior. El trabajo de $\operatorname{Rice}^{(34)}$ se centra también en otra medida de la espiritualidad, la Spiritual Comfort Measure (SCM), que aunque desarrollada en 2005(35), ha sido recientemente modificada para una mayor comprensión. Tal y como apunta Rice, la mayor novedad es la actualización de los anclajes, que en la nueva versión oscilan de 0 (confort completo) a 10 (dolor extremo). Sin embargo, no aparecen datos sobre la aceptación de la medida o de sus 


\section{Figura 1. Diagrama de flujo para la selección y evaluación de trabajos para la revisión.}

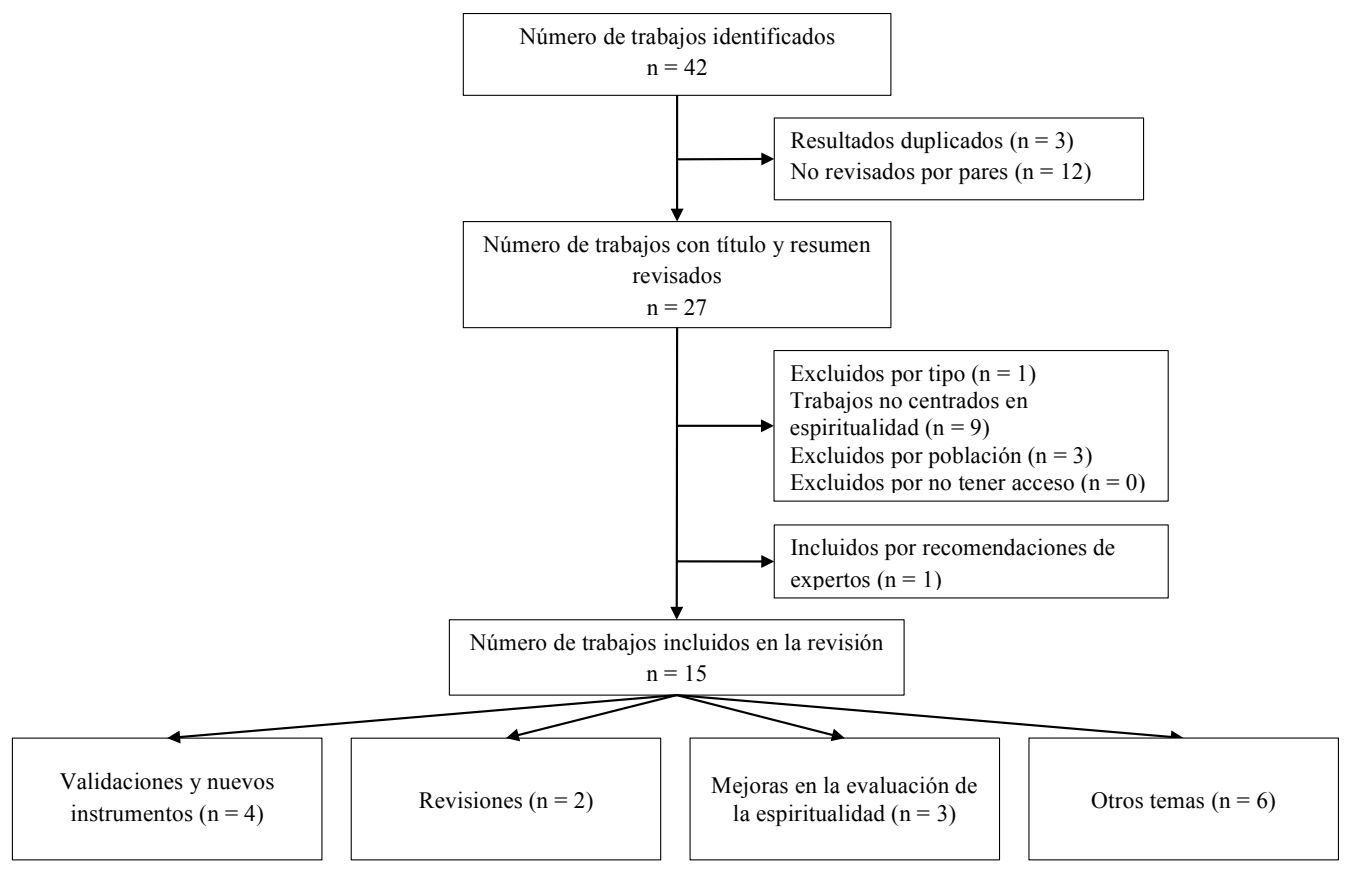

propiedades psicométricas. Rao, Salins, Deodhar y Muckaden ${ }^{(36)}$, por su parte, en lugar de ofrecer un instrumento concreto de medida de la espiritualidad, presentan un listado de preguntas dirigidas al paciente geriátrico oncológico. Este listado incluye dos preguntas para evaluar el estatus espiritual, a saber: " $¿$ Es la espiritualidad o la religión importante para ti?" y " $i$ Tus recursos espirituales están trabajando para ti?". De nuevo, no se ofrecen medidas de aceptabilidad, utilidad o propiedades psicométricas. El último trabajo en esta línea no presenta una nueva herramienta, sino que se centra en la evaluación de una ya existente y ampliamente usada, la Palliative Outcome Scale (POS), en su versión italiana ${ }^{(29)}$. En este caso, sin embargo, sí se ofrece información sobre algunas propiedades psicométricas relevantes, como la validez, la fiabilidad y la respuesta al cambio, ofreciendo resultados positivos.
Por lo que respecta a las revisiones encontradas sobre la espiritualidad y su evaluación, destacan dos trabajos. Por una parte, el trabajo de Best et al.(37), en el que se identifican un total de 11 medidas que evalúan el bienestar espiritual. Éstas varían en longitud, desde una simple pregunta, "¿Estás en paz?", como en el trabajo de Steinhauser et al. ${ }^{(38)}$, hasta escalas de mayor complejidad, que incluyen dimensiones de la propia espiritualidad, como es el caso de la Functional Assessment of Chronic IIIness Therapy-Spiritual WellBeing Scale (FACIT-Sp) ${ }^{(39-41)}$. Tras la comparación de dichas herramientas, los autores subrayan la FACIT-Sp como óptima para la evaluación del bienestar espiritual en el contexto oncológico, por haber sido desarrollada en una amplia población, su brevedad, su frecuencia de uso y los datos existentes sobre sus propiedades psicométricas y fácil interpretación ${ }^{(37)}$. En cuanto al 
segundo trabajo, éste se centra en analizar distintas aportaciones en el campo de la espiritualidad ${ }^{(42)}$. Una de las cuatro temáticas principales de los trabajos identificados es la que se centra en el desarrollo de instrumentos de evaluación de la dimensión espiritual en el ámbito de los cuidados paliativos. En este campo, Evangelista et al.(42), identificaron 73 instrumentos que de alguna forma estaban relacionados con la dimensión espiritual de pacientes de cuidados paliativos. De éstos, solo 9 resultaron validados transculturalmente, destacando herramientas como la FACIT-Sp o la POS. Los autores subrayan la necesidad de tener en cuenta las características clínicas y culturales de la población en la que originalmente se desarrolló el instrumento, así como sus propiedades psicométricas, aunque apuntan también que es una práctica poco habitual.

El tercer bloque de artículos encontrados se caracteriza por incluir trabajos que se centran en proponer mejoras en la evaluación de la espiritualidad. GómezCastillo et al.(43), por ejemplo, proponen un programa para mejorar la calidad de la evaluación de la espiritualidad, tras observar las bajas tasas de información espiritual que aparecen en el historial de un grupo de pacientes de cuidados paliativos. En esta línea, alientan a los profesionales a establecer una relación de confianza médico-paciente que permitiría a los pacientes compartir inquietudes espirituales con ellos. También recomiendan las visitas de profesionales en atención espiritual para promover la evaluación espiritual y atender sus necesidades en esta dimensión desde que son remitidos a cuidados paliativos. Currow et al. ${ }^{(44)}$, también estudian cómo mejorar la evaluación de las necesidades espirituales de estos pacientes, apostando por la evaluación en las distintas fases del cuidado y la recogida de información sistemática. Finalmente, el trabajo de Salsman et al. ${ }^{(45)}$, se centra en la necesidad de desarrollar un meta-análisis sobre el papel de la espiritualidad, dado que desde la perspectiva de su evaluación serviría para homogeneizar los resultados encontrados con las diversas medidas que se utilizan a lo largo de la literatura.

El último grupo de trabajos, se centra en distintos temas, más o menos relacionados con la evaluación de las necesidades espirituales. Tres de los trabajos incluidos hacen hincapié en la necesidad de evaluar la espiritualidad como base para conocer la eficacia de las intervenciones en los pacientes de cuidados paliativos ${ }^{(46-48)}$. Otros dos se centran en la evaluación de la dignidad, relacionándola con los recursos espirituales ${ }^{(49,50)}$. Y, finalmente, otro trabajo apunta la necesidad de evaluar la espiritualidad como predictor de la muerte asistida $^{(51)}$. En la tabla 1 pueden observarse las características de los artículos revisados.

\section{DISCUSIÓN/CONCLUSIONES}

El desarrollo de los cuidados paliativos y en general de una atención clínica más centrada en la persona, en el entorno de la enfermedad avanzada y terminal, ha llevado a una mejor comprensión de los aspectos subjetivos del proceso de enfermar y morir. De esta forma se ha ido despertando el interés por el sufrimiento, y cómo atender la dimensión espiritual o trascendente entendida ésta como un universal humano, que se caracteriza por ser transpersonal y transconfesional.

La tarea no es fácil, toda vez que la evaluación y acompañamiento espiritual se ven dificultadas por la propia naturaleza de esta dimensión, que se resiste a ser explorada por el paradigma experimental y es de difícil conceptualización ${ }^{(52)}$. También la dificulta la falta de consenso sobre modelos de espiritualidad en clínica, que ha llevado a una proliferación reciente de herramientas de evaluación. En muchas de ellas, si lo hay, no se explicita ni el modelo 


\begin{tabular}{|c|c|c|c|c|c|c|c|}
\hline 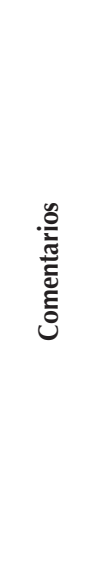 & 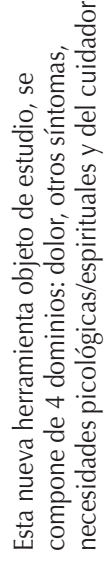 & & 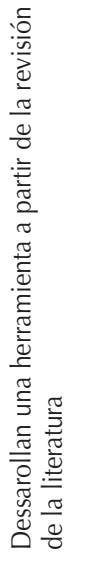 & 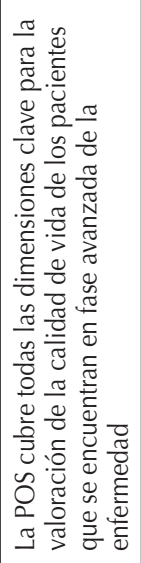 & 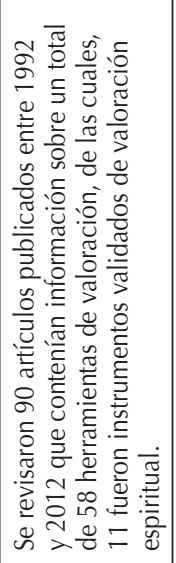 & 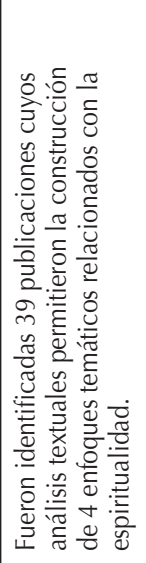 & 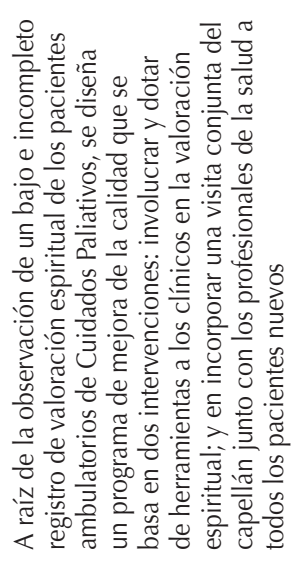 \\
\hline 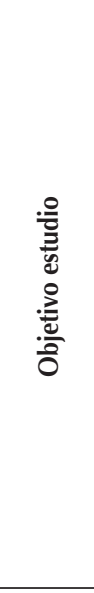 & 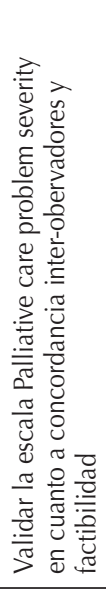 & 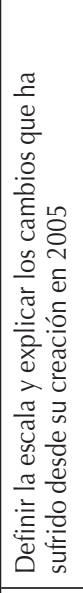 & 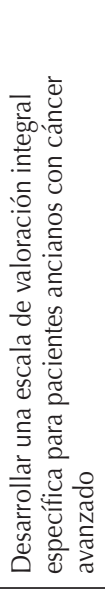 & 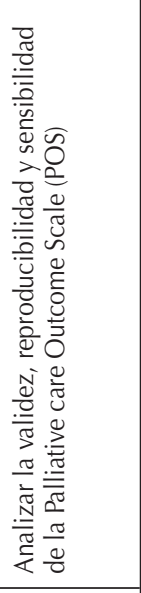 & 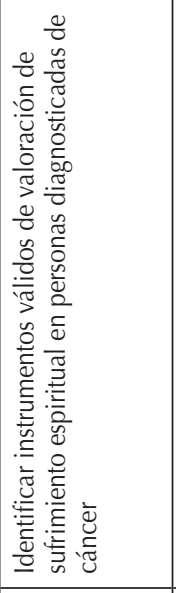 & 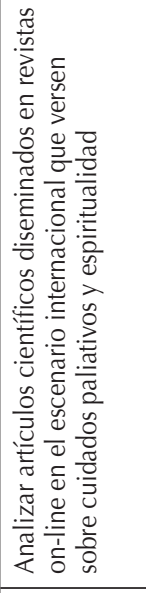 & 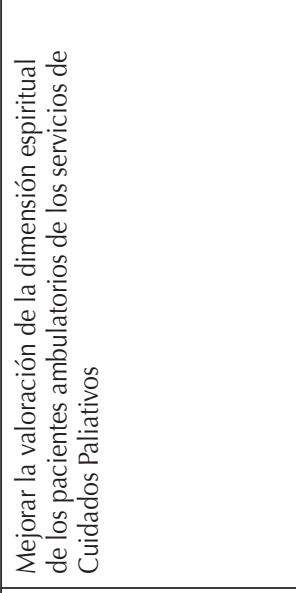 \\
\hline 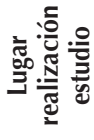 & 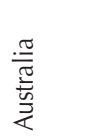 & 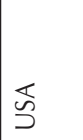 & 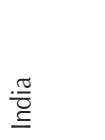 & $\frac{. \widetilde{T}}{\underline{I}}$ & 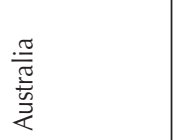 & $\mid \begin{array}{l}\overline{\bar{n}} \\
\overline{\frac{\pi}{n}}\end{array}$ & 六 \\
\hline 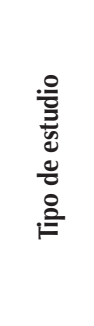 & 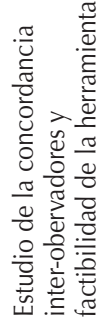 & 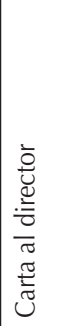 & 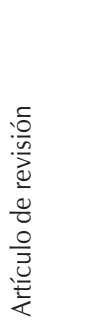 & 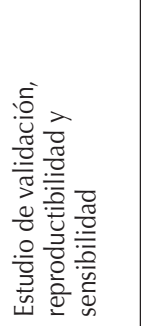 & 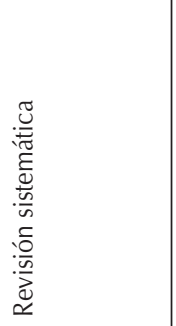 & 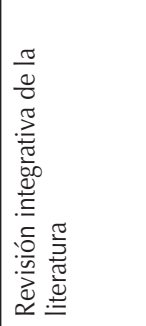 & 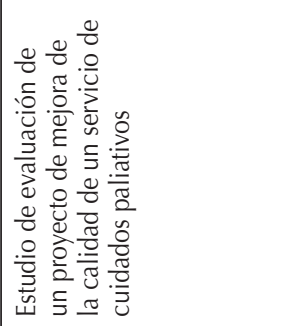 \\
\hline 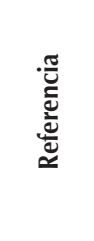 & 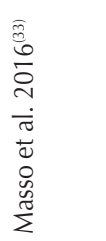 & 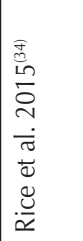 & 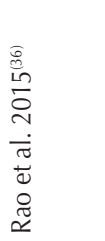 & 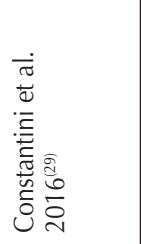 & 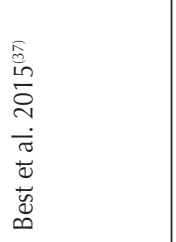 & 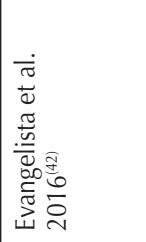 & 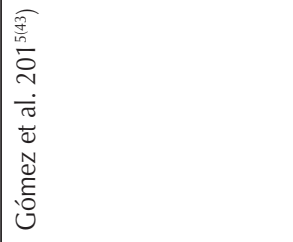 \\
\hline
\end{tabular}




\begin{tabular}{|c|c|c|c|c|c|c|c|}
\hline 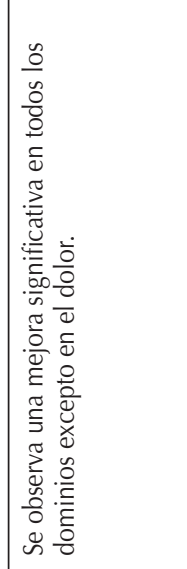 & 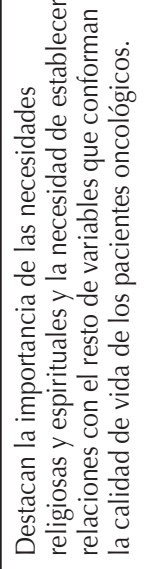 & 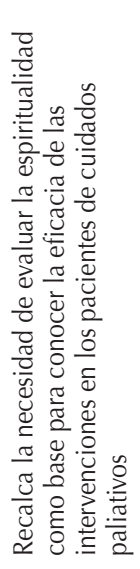 & & 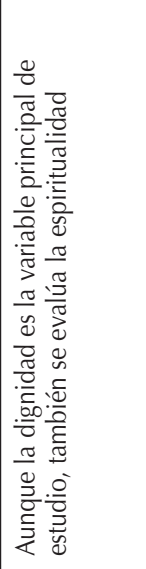 & 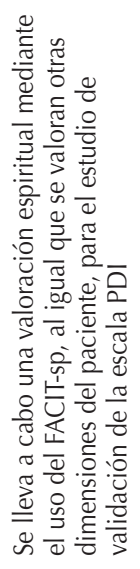 & 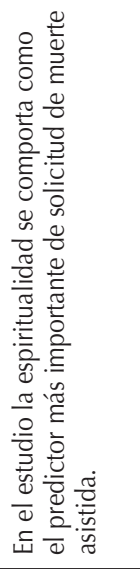 & 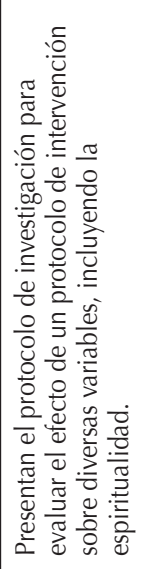 \\
\hline 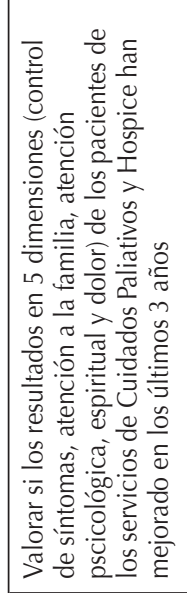 & 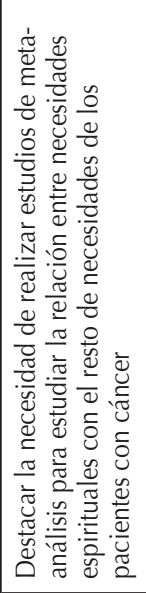 & 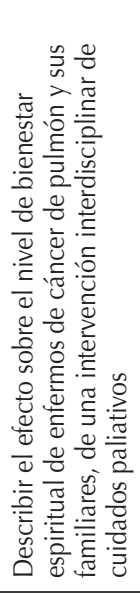 & 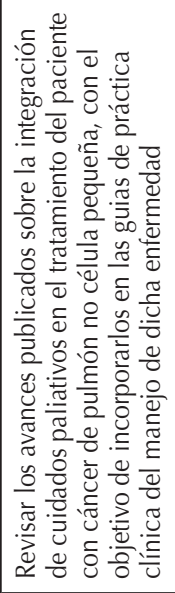 & 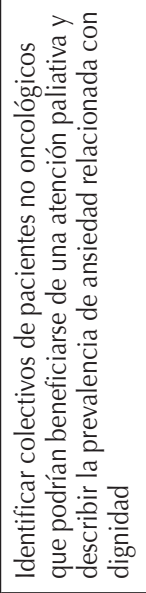 & 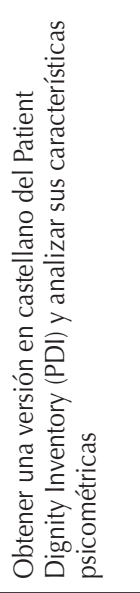 & 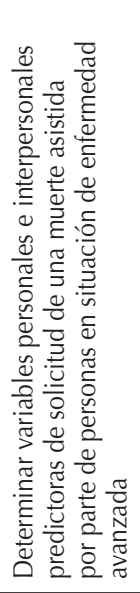 & 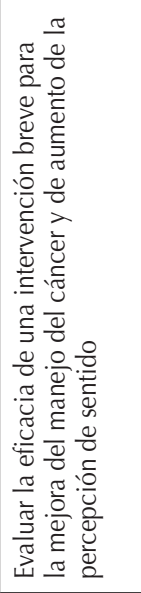 \\
\hline 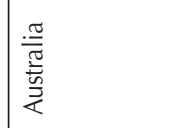 & 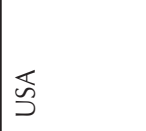 & 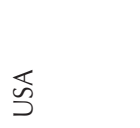 & 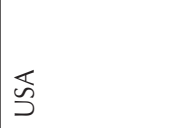 & 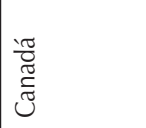 & 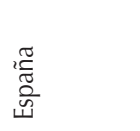 & 芯 & 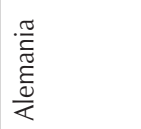 \\
\hline 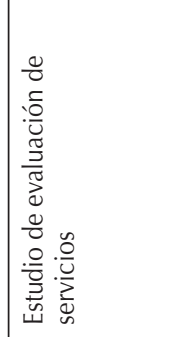 & 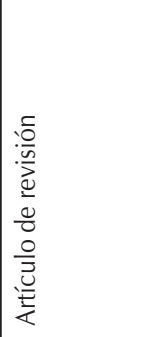 & 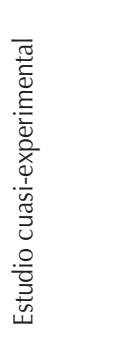 & 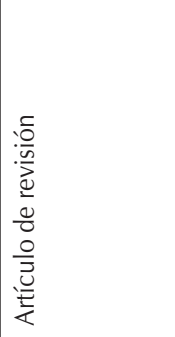 & 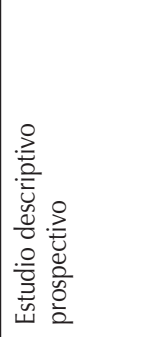 & 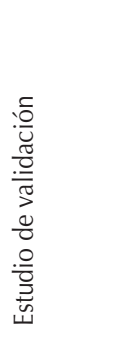 & 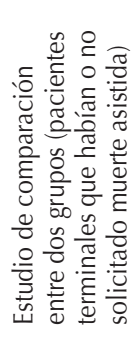 & 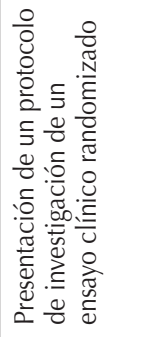 \\
\hline 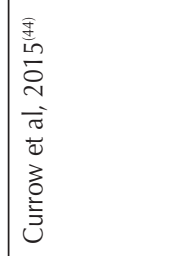 & 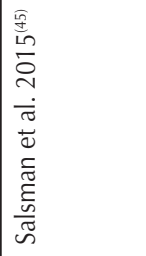 & 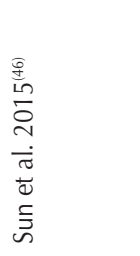 & 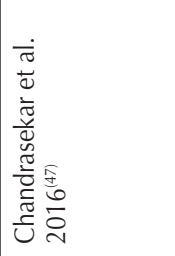 & 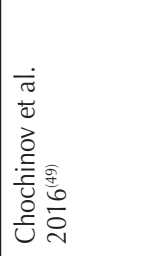 & 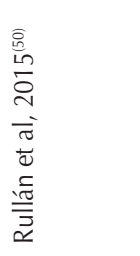 & 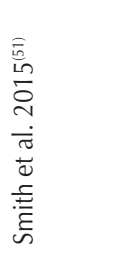 & 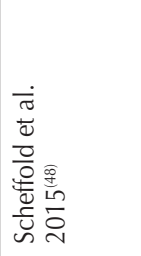 \\
\hline
\end{tabular}


conceptual subyacente ni sus propiedades psicométricas. La mayoría de cuestionarios se han desarrollado en población caucásica de Estados Unidos ${ }^{(1,28)}$ y apenas disponemos de validaciones transculturales de estos instrumentos.

La evaluación y acompañamiento espiritual están muy condicionados por el entorno cultural en que se da el proceso. Por ello, desde la SECPAL, partiendo de un modelo antropológico, que reconoce nuestra naturaleza espiritual y la persona como ser en relación, a través de su Grupo de Espiritualidad (GES), construyó un cuestionario basado en los recursos/necesidades de conexión en 3 niveles (intrapersonal, interpersonal y transpersonal). El objetivo era ayudar a explorar estos niveles en la biografía de cada paciente e identificar aquellas necesidades que necesitan ser atendidas/acompañadas, así como los recursos o fortalezas a reconocer y validar en cada paciente ${ }^{(5)}$. Posteriormente, se procedió a la validación del cuestionario GES adaptado a nuestro entorno y basado en el modelo citado ${ }^{(15)}$.

En 2014, una revisión crítica de los cuestionarios de evaluación e intervención espiritual disponibles en aquel momento $^{(30)}$, encontró 57 cuestionarios de los cuales tan solo 8 estaban validados transculturalmente y compartían la estructura del cuestionario GES que permite evaluar y atender las dimensiones intra, inter $y$ transpersonales de la espiritualidad.

En un ámbito de investigación tan emergente como éste, el presente estudio plantea los avances más recientes, en los últimos dos años. La revisión siguiendo la metodología propuesta por PRISMA ha permitido identificar en estos dos años, 42 estudios, ratificando el creciente interés por su estudio. De éstos se seleccionaron los 15 que cumplían los criterios demandados. Se han subclasificado según las características en 4 subgrupos: nuevos cuestionarios, revisiones, mejoras y otros temas. En el ámbito de nuevos cuestionarios los que han aparecido son de evaluación integral de necesidades entre las que genéricamente se exploran las espirituales. Este tipo de cuestionario está enfocado a identificar aquellos temas sobresalientes y por tanto es una aproximación más de screenning que de evaluación específica. Ninguno de ellos aporta información sobre criterios de complejidad que orienten a remitir a las personas a un referente para la atención específica, ni tampoco informan de su aceptabilidad ni medidas psicométricas. Otros son propuestas de mejora o validación de traducciones de cuestionarios conocidos. En general, las nuevas propuestas evalúan la espiritualidad como parte de una evaluación integral de las necesidades del paciente, y no se ha desarrollado ningún instrumento específico (el único específico es el de Rice $^{(34)}$, que no es nuevo, sino una modificación del ya existente).

Entre las revisiones de instrumentos de acompañamiento de este periodo encontramos dos artículos que coinciden en la amplia experiencia acumulada y la bondad de uso de la sub escala de bienestar espiritual de la Escala de Evaluación Funcional en enfermedades crónicas, conocida como FACIT-Sp (versión 7). Recoge aspectos del bienestar físico, social, emocional y funcional y finalmente presenta una serie de 12 preguntas bajo el epígrafe "Otras Preocupaciones" que aborda aspectos como el sentido y propósito en la vida, paz, fe y creencias espirituales. Los autores la proponen como óptima para la evaluación del bienestar espiritual en el contexto oncológico, por haber sido desarrollada en una amplia población, por su brevedad, su frecuencia de uso y los datos existentes sobre sus propiedades psicométricas y su fácil interpretación. Desde nuestra perspectiva tiene una carencia importante para ser usada en nuestro entorno cultural, al no explorar la dimensión inter- 
personal, las relaciones significativas y su armonía, que conocemos como un área donde las personas podemos tener -si las relaciones significativas con los allegados son armónicas y amorosas- un recurso importante para aceptar la realidad del proceso. Por otra parte esta dimensión puede ser fuente de distrés y sufrimiento si hay relaciones conflictivas o asuntos pendientes que dificulten la partida. Conociendo la importancia de las relaciones familiares en nuestro entorno social, tanto en España como en Latino América creemos necesario que cualquier acompañamiento explore los recursos y necesidades del ámbito interpersonal.

El último grupo de artículos aportan indicaciones para profundizar en la comprensión y aplicación del cuidado y acompañamiento espiritual en esta etapa de la vida.

Según nuestra visión, todas estas aportaciones, herramientas, cuestionarios son únicamente esquemas, apoyos a los profesionales para una aproximación a la realidad clínica en mapas que nos permiten acercarnos a acompañar el viaje de la persona en proceso de enfermedad terminal y en la proximidad de la muerte. El mapa nunca debe confundirse con el territorio y, cabe recordar, que el mejor guía no es quien ha leído muchos mapas sino quien mejor conoce el territorio por la experiencia personal de haber elaborado las propias pérdidas y por la experiencia de haber atendido, acompañado y aprendido de nuestros mejores maestros, que son siempre nuestros pacientes.

\section{REFERENCIAS BIBLIOGRÁFICAS}

* los artículos marcados con asteriscos son los incluidos en la revisión

1. Selman L, Harding R, Gysels M, Speck P, Higginson IJ. The measurement of spirituality in palliative care and the content of tools validated cross-culturally: A sys- tematic review. J Pain Symptom Manage 2011;41:728-53. Doi: 10.1016/j.jpainsymman.2010.06.023.

2. Vachon M, Fillion L, Achille M. A conceptual analysis of spirituality at the end of life. J Palliat Med 2009;12:53-9. Doi: 10.1089/jpm.2008.0189.

3. National Consensus Project for Quality PaIliative Care. Clinical practice guidelines for quality palliative care. 2011. [Accesso 11 de Junio 11, 2014].

Disponible en: http://www.nationalconsensusproject.org/guideline.pdf.

4. National Quality Forum. A national framework and preferred practices for palliative and hospice care: A Consensus Report. Washington, DC: National Quality Forum.

5. Benito E, Barbero J, Dones M. Espiritualidad en clínica. Una propuesta de evaluación y acompañamiento Espiritual en Cuidados Paliativos. 2014 Madrid: Sociedad Española de Cuidados Paliativos.

6. Nolan S, Saltmarch P, Leget C. Spiritual care in palliative care: Working towards an EAPC Task Force. Eur J Pal Care 2011;18: 86-89.

7. Puchalski C. M,Vitillo R, Hull SK, Reller $N$. Improving the spiritual dimension of whole person care: Reaching national and international consensus. J Pall Med 2014;17:1-15.

8. National Comprehensive Cancer Network. Guidelines version 2 2013. Distress management. [Acceso 15 de octubre de 2016] Disponible en: http://wwwnccn.org/professionals/phsician-gls/pdf/palliative.pdf. Accessed May 6, 2013.

9. National Consensus Project. Clinical practice guidelines 2013. Available at: http:// www.nationalconsensusproject.org/NCP. Accessed May 6, 2013.

10. Guía de Espiritualidad en clínica. Sociedad Española de Cuidados Paliativos (SECPAL), 2008.

11. Pearce MJ, Coan AD, Herndon JE, Koenig HG, Abernethy AP. Unmet spiritual care 
needs impact emotional and spiritual wellbeing in advanced cancer patients. Support Care Cancer 2012; 20:2269-2276. Doi: 10.1007/s00520-011-1335-1

12. Winkelman WD, Lauderdale K, Balboni MJ, Phelps AC, Peteet JR, Block SD, et al. The relationship of spiritual concerns to the quality of life of advanced cancer patients: preliminary findings. J Palliat Med 2011;14:1022-8. Doi: 10.1089/jpm.2010.0536

13. Balboni TA, Vanderwerker LC, Block SD, Paulk ME, Lathan CS, Peteet JR, et al.. Religiousness and spiritual support among advanced cancer patients and associations with end-of-life treatment preferences and quality of life. J Clin Oncol 2007;25:55560. Doi: 10.1200/JCO.2006.07.9046

14. Barreto P, Fombuena M, Diego R, Galiana L, Oliver A, Benito E. Bienestar emocional y espiritualidad al final de la vida. Med Paliativ 2015;22:25-32.

15. Benito E, Oliver A, Galiana L, Barreto P, Pascual A, Gomis C, et al. Development and validation of a new tool for the assessment and espiritual care of palliative care patients. J Pain Symptom Manage 2014;47:1008-18. Doi: 10.1016/j.jpainsymman.2013.06.018.

16. Cannon AJ, Darrington DL, Reed EC, Loberiza FR. Spirituality, patients' worry, and follow-up health-care utilization among cancer survivors. J Support Oncol 2011;9:141-8. Doi:10.1016/j.suponc.2011.03.00

17. Ferrell B, Otis-Green S, Economou D. Spirituality in cancer care at the end of life. Cancer J 2013;19:431-7. Doi: 10.1097/ PPO.0b013e3182a5baa5

18. Peteet JR, Balboni MJ. Spirituality and religion in oncology. CA Cancer J Clin. 2013;63:280-9. Doi: 10.3322/caac.21187.

19. Puchalski C, Ferrell B, Virani R, Otis-Green S, Baird P, Bull J, et al. Improving the quality of spiritual care as a dimension of paIliative care: the report of the Consensus Conference. J Palliat Med 2009;12:885904. Doi: 10.1089/jpm.2009.0142.
20. Visser A, Garssen B, Vingerhoets A. Spirituality and well-being in cancer patients: a review. Psychooncology 2010; 19: 56572. Doi: 10.1002/pon.1626.

21. Balboni TA, Paulk ME, Balboni MJ, et al. Provision of spiritual care to patients with advanced cancer: Associations with medical care and quality of life near death. J Clin Oncol 2010;28:445-52. Doi: 10.1200/JCO.2009.24.8005.

22. Balboni T, Balboni M, Paulk ME, Phelps AC, Loggers ET, Wright AA, et al. Support of cancer patients' spiritual needs and associations with medical care costs at the end of life. Cancer 2011;117:5383-91. Doi: 10.1200/JCO.2009.24.8005

23. Park CL, Cho D, Blank TO, Wortmann JH. Cognitive and emotional aspects of fear of recurrence: predictors and relations with adjustment in young to middle-aged cancer survivors. Psychooncology 2013; 22:1630-8. Doi: 10.1002/pon.3195

24. Park C, Edmondson D, Hale-Smith A, Blank T. Religiousness/spirituality and health behaviors in younger adult cancer survivors: Does faith promote a healthier lifestyle? J Behav Med 2009;32:582-91. Doi: 10.1007/s10865-009-9223-6.

25. Hodge DR. A Template for spiritual assessment: A review of the JCAHO requirements and guidelines for implementation. Social Work 2006;51:317-326.

26. Ramondetta L, Sun C, Surbone A, Olver I, Ripamonti C, Konishi T, et al. Surprising results regarding MASCC members' beliefs about spiritual care. Support Care Cancer 2013;21:2991-8. Doi: 10.1007/s00520013-1863-y.

27. Dones M, Collette N, Barbero J, Gomis C, Balbuena P, Benito E. ¿Cómo percibimos los profesionales el acompañamiento espiritual en Cuidados Paliativos en España? MEDIPA 2016;23:63-71

28. Oliver A, Galiana L, Benito E. Evaluation tools for spiritual support in end of life care: increasing evidence for their clinical application. Curr Opin Support Pa- 
Iliat Care 2016;9:357-60. Doi: 10.1097/ SPC.0000000000000173.

29. ** Costantini M, Rabitti E, Beccaro M, Fusco F, Peruselli C, La Ciura P, Valle A, Suriani $C$, et al. Validity, reliability and responsiveness to change of the Italian palliative care outcome scale: A multicenter study of advanced cancer patients. BMC PalliatCare 2016;15:23. Doi: 10.1186/s12904-0160095-6

30. Galiana L, Oliver A, Gomis C, Barbero J, Benito E. Cuestionarios de evaluación e intervención espiritual en cuidados paliativos: una revisión crítica. Med Paliativ 2014;21:62-74.

31. Hill PC, Hood RWJ. Measures of religiosity. 1999. Birmingham, AL: Religious Education Press.

32. Moher D, Liberati A, Tetzlaff J, Altman DG; PRISMA Group. Preferred reporting items for systematic reviews and meta-analyses: the PRISMA statement. Ann Intern Med 2009;151:264-9. Doi: 10.7326/00034819-151-4-200908180-00135

33. **Masso $\mathrm{M}$, Allingham SF, Johnson $\mathrm{CE}$, Pidgeon T, Yates P, Currow D, et al. PaIliative care problem severity score: Reliability and acceptability in a national study. Palliat Med 2016;30:479-85 Doi: 10.1177/0269216315613904.

34. **Rice L. The Spiritual comfort measure. J Palliat Med 2015;18:910-1. Doi: 10.1089/ jpm.2015.0262.

35. Cook N, Klauer R, Spencer R, et al. The Spiritual comfort measure. The Center for Hospice and Palliative Care, 2005.

36. **Rao S, Salins N, Deodhar J, Muckaden M. Developing a comprehensive cancer specific geriatric assessment tool. Indian J Cancer 2015;52:94-7. Doi: 10.4103/0019509X.175588.

37. ** Best M, Aldridge L, Butow P, Olver I, Price M, Webster F. Assessment of spiritual suffering in the cancer context: A systematic literature review. Palliat Support Care 2015;13:1335-61. Doi: 10.1017/ S1478951514001217.
38. Steinhauser K, Voils Cl, Clipp EC, Bosworth HB, Christakis NA, Tulsky JA. Are you at peace?: One item to probe spiritual concerns at the end of the life. Arch Intern Med 2006;166:101-5. Doi:10.1001/ archinte.166.1.101

39. Canada AL, Murphy PE, Fitchett G, Peterman $\mathrm{AH}$, Schover LR. A three-factor model for the FACIT-Sp. Psychooncology 2008;17:908-16. Doi: 10.1002/pon.1307

40. Murphy PE, Canada AL, Fitchett G, Stein K, Portier K, Crammer C, Peterman AH. An examination of the 3-factor model and structural invariance across racial/ethnic groups for the FACIT-Sp: A report from the American Cancer Society's Study of Cancer Survivors - II (SCS - II). Psychooncology 2010; 19:264-72. Doi: 10.1002/ pon.1559

41. Peterman AH, Fitchett G, Brady MJ, Hernandez, Cella D. Measuring spiritual wellbeing in people with cancer: The functional assessment of chronic illness therapy. Spiritual Well-Being Scale (FACIT-Sp). Ann Behav Med 2002;24:49-58.

42. **Evangelista CB, Lopes MEL, Costa SFG, Batista PSS, Batista JBV, et al. Palliative care and spirituality: an integrative literature review. Rev Bras Enferm [Internet]. 2016;69:554-63. Doi:10.1590/00347167.2016690324i.

43. **Gomez-Castillo BJ, Hirsch R, Groninger $\mathrm{H}$, Baker K, Cheng MJ, Phillips J, et al. Increasing the number of outpatients receiving spiritual assessment: a pain and palliative care service quality improvement project. J Pain Symptom Manage 2015;50:724-9. Doi: 10.1016/j.jpainsymman.2015.05.012.

44. **Currow DC, Allingham S, Yates P, Johnson C, Clark K, Eagar K. Improving national hospice/palliative care service symptom outcomes systematically through point-of-care data collection, structured feedback and benchmarking. Support Care Cancer 2015;23:307-15. Doi: 10.1007/ s00520-014-2351-8. 
45. ** Salsman JM, Fitchett G, Merluzzi TV, Sherman AC, Park CL. Religion, spirituality, and health outcomes in cancer: A case for a meta-analytic investigation. Cancer. 2015;121:3754-9. Doi: 10.1002/ cncr.29349.

46. **Sun V, Kim JY Irish TL, Borneman T, Sidhu RK, Klein L, Ferrell B. Palliative care and spiritual well-being in lung cancer patients and family caregivers. Psychooncology 2015. Doi: 10.1002/pon.3987. [Epub ahead of print]

47. **Chandrasekar D, Tribett E, Ramchandran K. Integrated Palliative Care and Oncologic Care in Non-Small-Cell Lung Cancer. Curr Treat Options Oncol 2016;17:23. Doi: 10.1007/s11864-016-0397-1.

48. ** Scheffold K, Philipp R, Engelmann D, Schulz-Kindermann F, Rosenberger C, Oechsle K, et al. Efficacy of a brief manualized intervention Managing Cancer and Living Meaningfully (CALM) adapted to German cancer care settings: study protocol for a randomized controlled trial. BMC Cancer 2015;15:592. Publis- hed online 2015 Aug 19. Doi: 10.1186/ s12885-015-1589-y.

49. **Chochinov HM, Johnston W, McClement SE, Hack TF, Dufault B, Enns M, et al. Dignity and distress towards the end of life across four non-cancer populations. PLoS One. 2016;11:e0147607. Doi: 10.1371/ journal.pone.0147607. eCollection 2016.

50. **Rullán M, Carvajal A, Núñez-Córdoba JM, Martínez M, Carrasco JM, García I, et al. Spanish version of the Patient Dignity Inventory: Translation and validation in patients with advanced cancer. J Pain Symptom Manage 2015;50:874-81.e1. Doi: 10.1016/j.jpainsymman.2015.07.016. Epub 2015 Sep 3.

51. **Smith KA, Harvath TA, Goy ER, Ganzini L. Predictors of pursuit of physicianassisted death. J Pain Symptom Manage. 2015;49:555-61. Doi: 10.1016/j.jpainsymman.2014.06.010.

52. Sinclair S, Pereira J, Raffin S. A thematic review of the spirituality literature within palliative care J Palliat Med 2006; 9:46479. Doi:10.1089/jpm.2006.9.464. 
